\title{
Measuring the cryogenic optical alignment between the telescope element and the instruments module of the James Webb Space Telescope
}

\author{
Tony Whitman and Eugene Olczak, ITT Geospatial Systems
}

\begin{abstract}
The alignment between the Aft Optical Subsystem (AOS) and the Integrated Science Instruments Module (ISIM) is nonadjustable in orbit, so the alignment must be carefully verified in a cryogenic vacuum environment prior to launch. Optical point source locations calibrated by optical metrology instruments are imaged through the AOS onto the Science Instruments to determine focal, lateral, and clock angle alignment. The pupil image of the AOS is overlaid onto the pupil image of the NIRCam to determine the tip and tilt alignment. In addition, an image from fiducial lights at the Primary Mirror checks the pupil alignment between the telescope entrance pupil, the telescope pupil mask, and the NIRCam aperture stop. The image positions are combined to determine the relative alignment between the Optical Telescope Element (OTE) and the ISIM in all six degrees of freedom with corresponding alignment uncertainties. Uncertainties in the position of focused images of the test sources and images from the pupils are derived from sensitivities of an optical model of the system and the Science Instrument sensing capability. Additional uncertainty in the pupil alignment measurement is due to uncertainty in the analytical removal of gravity effects that simulate the onorbit alignment environment.
\end{abstract}

Keywords: JWST, optical, pupil, focus, alignment, cryogenic, telescope, detector

\section{INTRODUCTION}

The James Webb Space Telescope (JWST) shown in Figure 1 is a three mirror anastigmat (TMA) where the primary mirror (PM) and secondary mirror (SM) positions are adjustable in orbit. However, the tertiary mirror (TM) within the Aft Optical Subsystem (AOS) and the imaging Integrated Science Instrument Module (ISIM) are either non-adjustable or have limited range of adjustability ${ }^{1}$. As a result, the optical alignment between the AOS and the ISIM must be verified prior to launch. An important aspect of the alignment verification is the measurement of the alignment in a cryogenic vacuum environment.

One way to align two imaging systems is to coincide the focal surfaces and to coincide the exit pupil of the leading optical system with the entrance pupil of the trailing optical system as schematically shown in Figure 2. This is the method chosen to optically test the alignment between the AOS and the ISIM.

The exit pupil for the OTE is near a mask on the Fine Steering Mirror (FSM) along the optical path beyond the TM. The OTE forms a single $\sim 3$-meter radius spherical image surface with the (FSM) at the spherical center. Each Science Instrument (SI) picks off a portion of the OTE field with a fold mirror, then re-images that portion of the field to the instrument detectors such that the SI's entrance pupil is 3 meters ahead of the SI's object surface. The object surfaces of the SI's are aligned to geometrically match the OTE focal surface curvature and mechanically spaced at the OTE-toISIM interface to coincide with the designed OTE focal surface location. Likewise the entrance pupil for each SI is aligned to overlap the other SI's at the designed OTE exit pupil location. The location of the SI's relative to each other and the location of each SI relative to the OTE-to-ISIM interface is tested at the ISIM level of assembly.

After integrating the OTE to the ISIM to become the OTIS, a star scene can be imaged to all the Science Instruments to check the focus and lateral positions of the SIs and OTE. However, only the Near Infrared Camera (NIRCam) and the Mid-Infrared Imager (MIRI) have pupil imaging capabilities. Given the tested alignment of the SIs at the ISIM level of assembly, only one instrument, NIRCam, is necessary to measure the relative pupil alignment between the ISIM and the OTE. The other instrument, MIRI, can be used as further confirmation.

22 CFR 125.4 (b)(13) applicable. 


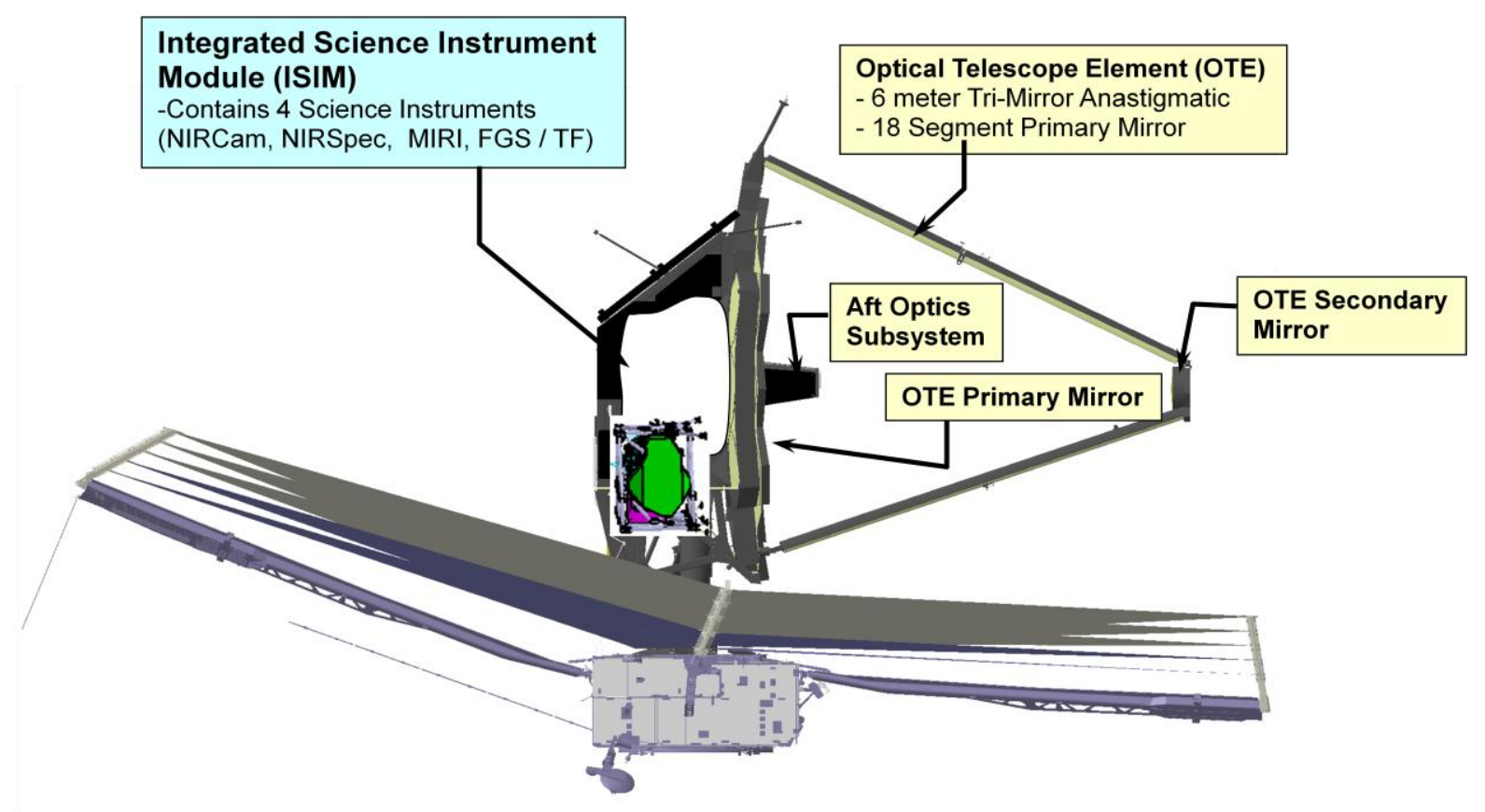

Figure 1 JWST architecture.

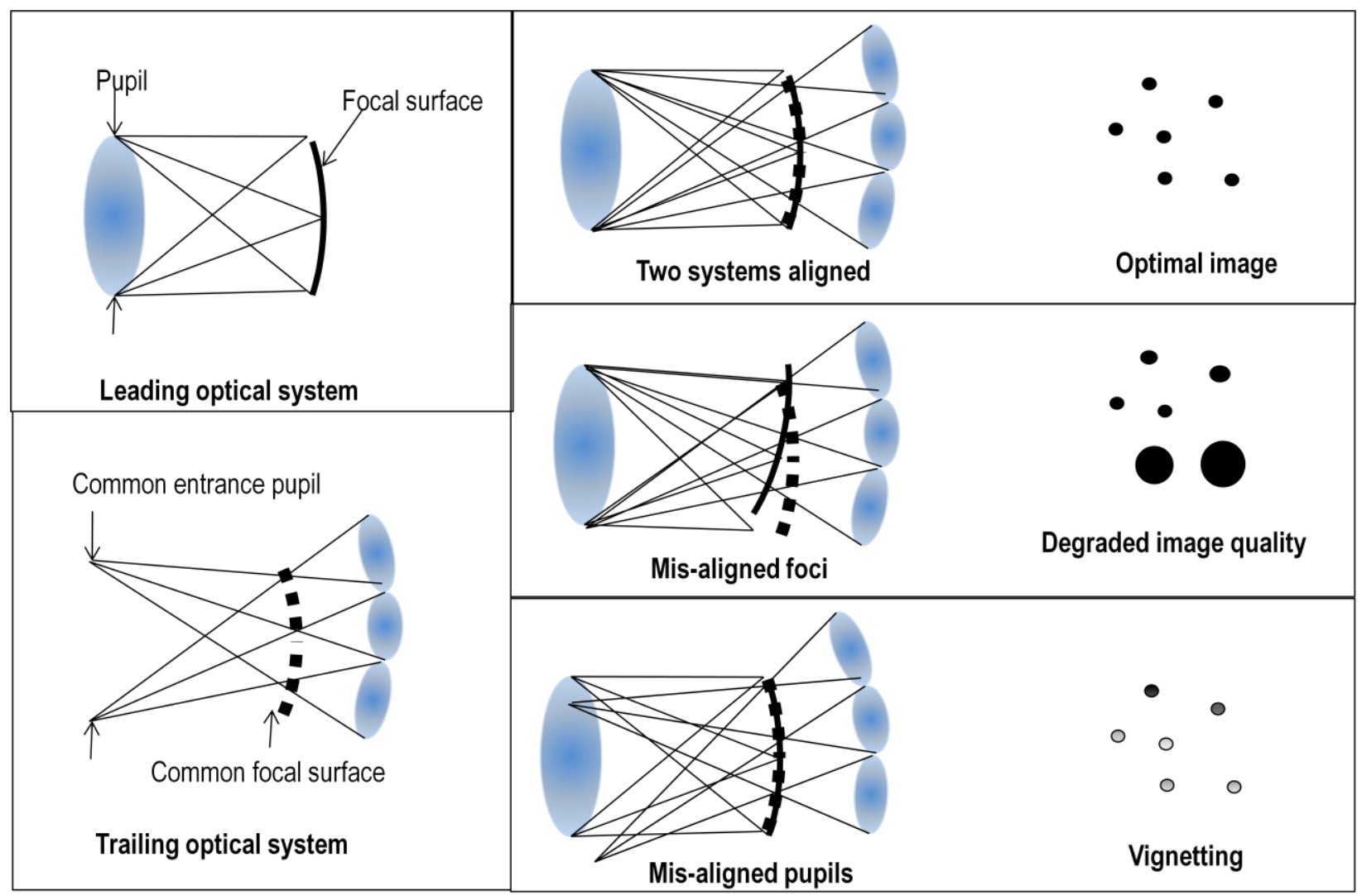

Figure 2 Illustrating the OTE as a singlet and the ISIM as a curved array of singlets, the optimally aligned combination has the pupils and focal surface coincide as shown in the upper right schematic. Mis-aligned focal surface leads to degraded image quality, especially with defocused images. Mis-aligned pupils principally lead to vignetting.

$$
22 \text { CFR } 125.4 \text { (b)(13) applicable. }
$$




\section{TEST DESCRIPTION}

\subsection{Focal surface alignment test}

Optical point sources will be placed at the intermediate, or Cassegrain, focal surface of the OTE located near the entrance aperture of the AOS in five locations spread across the OTE field of view approximately at the locations shown in Figure 3. (The sources are removed from the OTIS after this ground test.) The TM will image these sources to the common ISIM object surface. The image positions of the test sources relative to the AOS optical system are calibrated at the AOS level of assembly in a cryogenic test.

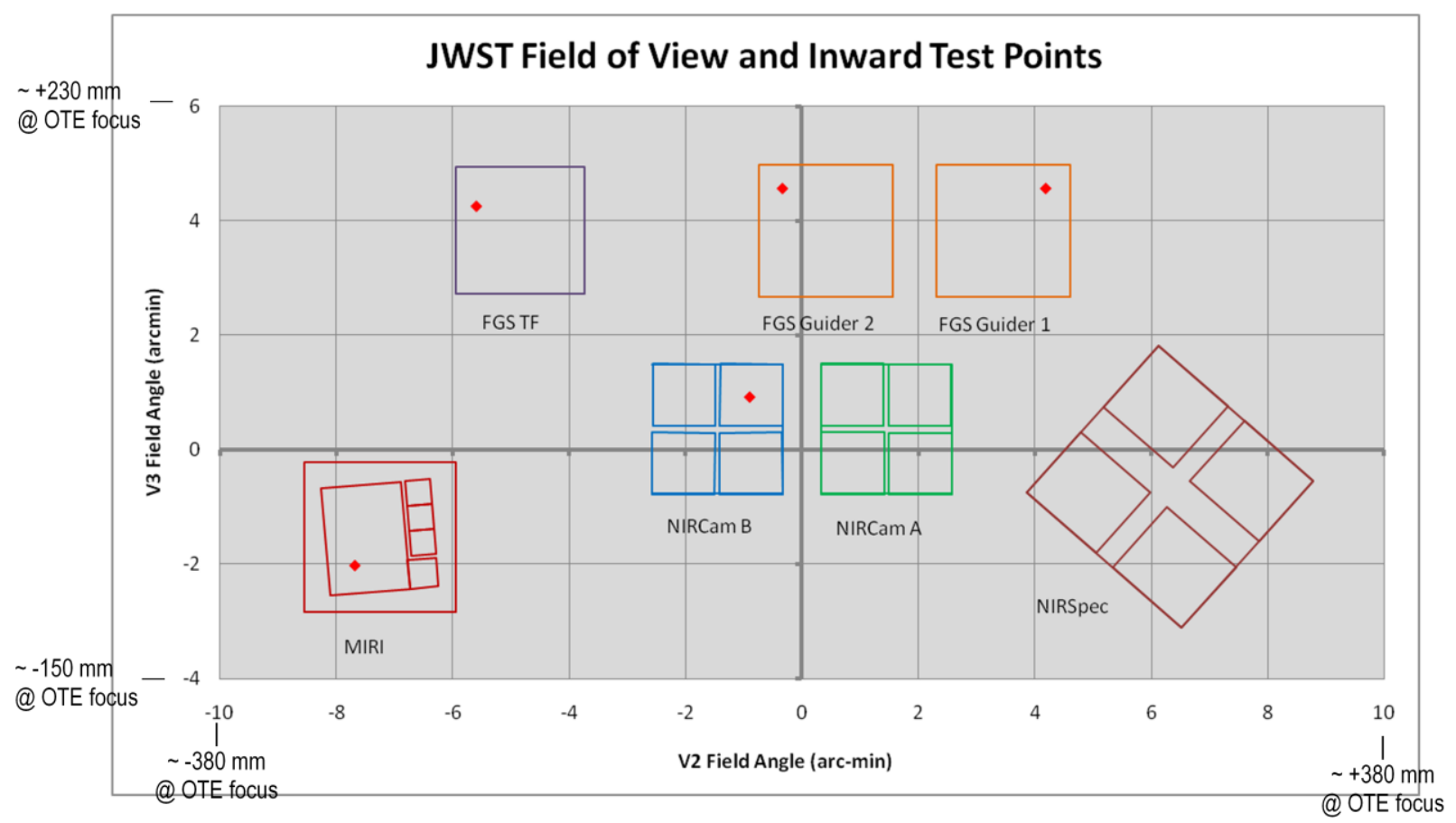

Figure 3 Approximate field map of test sources, designated by red diamonds, emitting into the AOS. $10 \operatorname{arcmin} \approx 380 \mathrm{~mm}$ at the OTE focal surface.

In the OTIS cryogenic test, the centroid position of test source images in the SI field is compared with the calibrated field position from the AOS cryogenic test to compare the SI detector position relative to the AOS laterally (decenter position and clocking angle) $)^{2}$.

Furthermore, the quantity of defocus in the test source images determines the relative focus between the AOS and the ISIM at the respective field positions.

\subsection{Pupil alignment test}

Three pupils are represented onto the NIRCam detector using the Pupil Imaging Lens in the NIRCam: the NIRCam aperture stop, the FSM mask in the AOS, and the OTE Primary Mirror. The relative lateral position (decenter and clocking of the pupils) and the relative diameters in the detector image are compared to determine pupil alignment.

\subsection{NIRCam aperture stop}

The NIRCam has a mechanized rotating wheel of optical components and a clear opening at the aperture stop location for the NIRCam. One component is an array of pinhole lights that image through the PIL to the detector. The array represents the lateral aperture stop position and the size of the aperture stop.

\subsection{FSM mask}

The FSM mask is illuminated by one of the Cassegrain optical test sources. With the NIRCam PIL inserted the illuminated mask near the OTE exit pupil is imaged onto the detector. See Figure 6. 


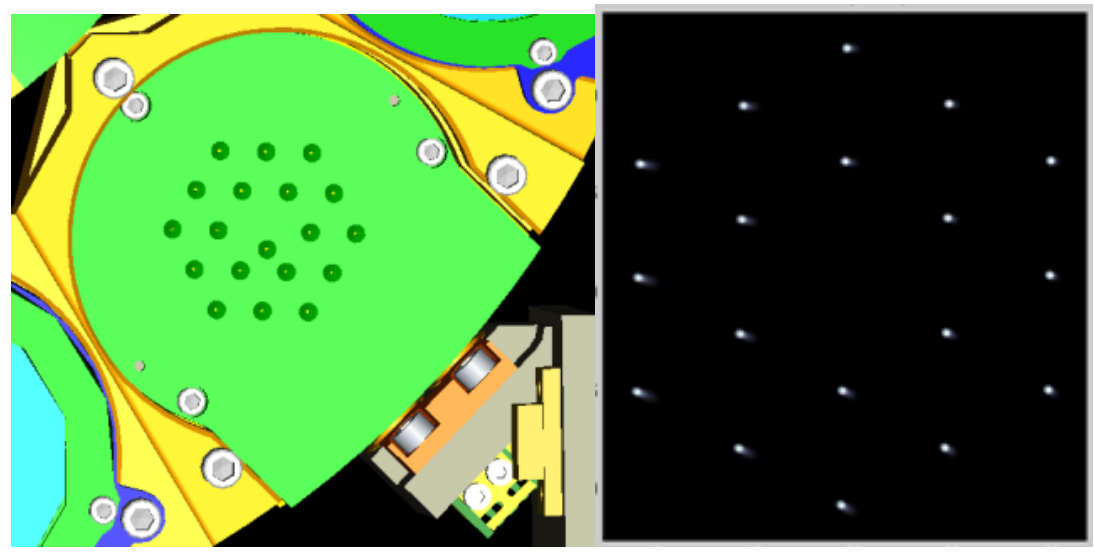

Figure 4 Drawing of pinhole light array in NIRCam and simulated image of array
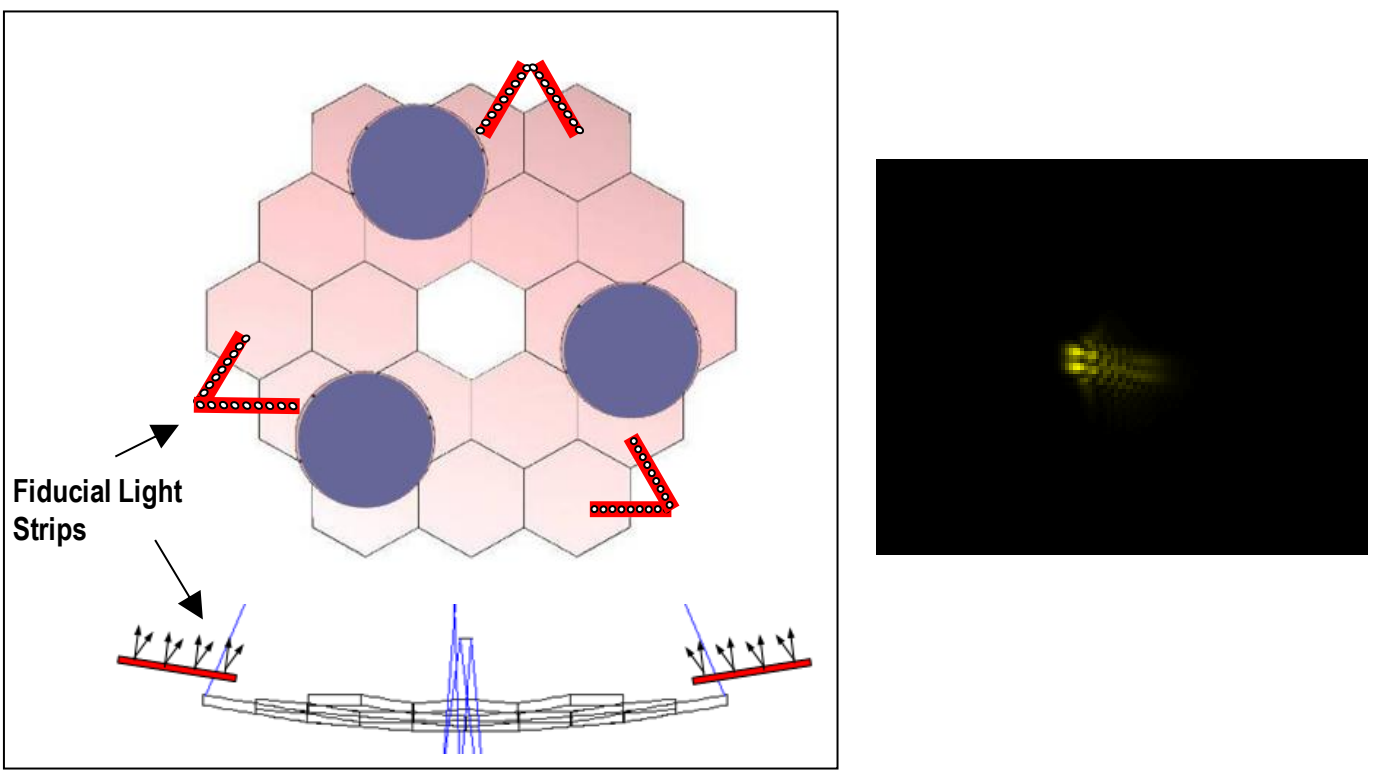

Figure 5 Schematic of linear arrays of point sources near PM and a sample simulated image from 2 adjacent sources.
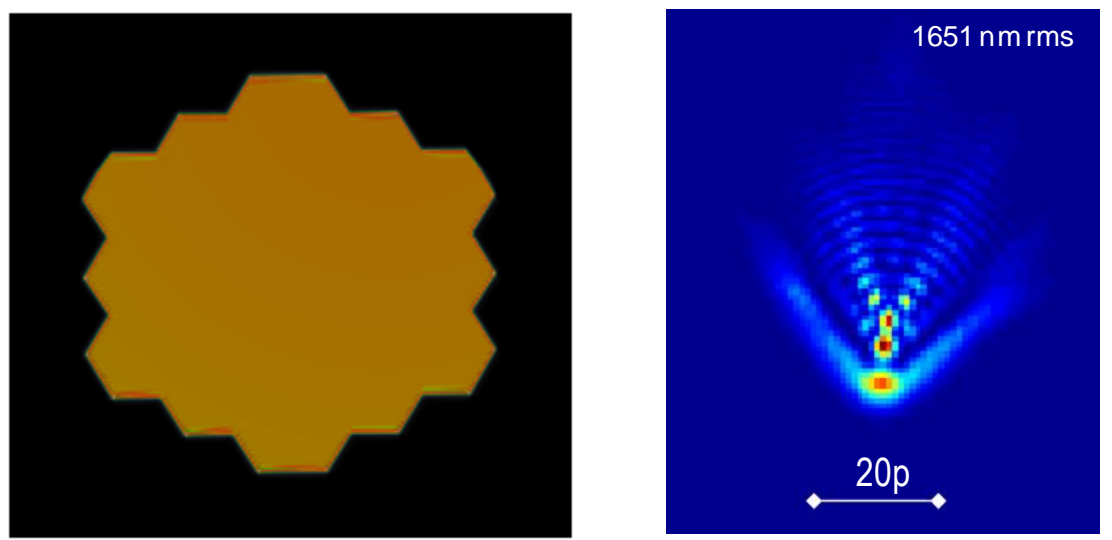

Figure 6 Simulated FSM mask image (Courtesy from NASA) and simulated NIRCam image from inward Cassegrain point source 


\subsection{Primary Mirror}

In addition, linear arrays of optical point sources are positioned radially outward near the PM, the entrance pupil of the OTE. The light from these arrays reflect off the OTE Secondary Mirror into the AOS to the NIRCam detector. The lights are individually addressable and their positions are registered with respect to the PM. If the light from one of these point sources passes through to the NIRCam detector, then the point source image is inside the FSM mask opening. If the light from the point source is blocked from reaching the NIRCam detector, then the point source image is outside the FSM mask opening. The edge of the mask therefore maps to the position between the passed point source and the blocked point source on each linear array. Since the point sources are registered to the edge of the PM using metrology, the PM lateral position and diameter relative to the FSM mask is determined.

The image location of these point sources on the NIRCam detector with the PIL inserted also checks the alignment of the FSM mask.

\section{DETERMINING ALIGNMENT BETWEEN THE ISIM AND THE OTE}

\subsection{De-center ( $x$ and $y$ translation)}

The de-center position of the ISIM relative to the OTE is a simple 1:1 correspondence of the test source image centroid relative to the detector pixel locations. Each source provides a de-center value, so 5 points provides additional information on the statistical distribution and relative SI positions since the points are spread among 3 SIs.

The uncertainty in an individual test source de-center value is dominated by the calibration uncertainty of the image location relative to the AOS coordinate references in the AOS cryogenic test which includes the image centroiding uncertainty, an uncertainty that also occurs in the OTIS cryogenic test images. Due to the off-axis design of the OTE, point sources from the Cassegrain focus surface create highly aberrated images (Figure 6) adding to the complexity of the centroiding algorithm.

Furthermore, a significant error contribution comes from the repeatability of mounting the optical test source onto the AOS. After calibration in the AOS cryogenic test, the test sources must be removed for shipment and mechanical environmental testing of the AOS and OTIS.

Nonetheless, the uncertainty for determining the decenter of an SI detector pixel location relative to the AOS coordinate system is still under 1/3 of a millimeter. Each pixel of the Fine Guidance Sensor detector, for example, corresponds to $\sim 50$ micrometers at the OTE focal surface, so $1 / 3 \mathrm{~mm}$ equates to 7 pixels.

\subsection{Clocking (rotation about the z-axis)}

The clocking rotation of the ISIM relative to the OTE is determined by measuring the relative rotation of the line segment between two test images and the line segment between the two corresponding detector pixel locations. With 5 test points, this leads to 10 pair combinations to generate clock angle values. The angular direction of the line segments is calculated using the endpoint (image or detector pixel) decenter position and the distance between the endpoints.

The angular direction uncertainty is dominated by the de-center uncertainty in section 3.1 with enhancement from the systematic errors that double with two points in the calculation.

Note that the field points are located far apart across the field to maximize the sensitivity of the measurement - in other words, to increase the length of the lever arm between the two endpoints.

Ultimately, the clocking uncertainty of a single pair of points across the field is expected to be less than 1.5 milliradians.

\subsection{Focus (z translation)}

Each tested field point actually has multiple point sources at varied focal positions. This configuration allows retrieval of the phase information from the image point spread functions. With phase retrieval, the wavefront error of the image can be mapped. Then the detector position relative to the test image focal position can be determined by extracting the power component of the wavefront error ${ }^{2}$. 
The telescope has an F-number equal to 20, resulting in a large depth of focus. Most of the uncertainty in determining focus location comes from the uncertainty determining the power component of the wavefront error and the insensitivity of the power wavefront error relative to focal position. This uncertainty occurs in both the calibration of the focal position in the AOS cryogenic test and the measurement during the OTIS cryogenic test.

Another significant error comes from the uncertainty of the sag in the ISIM due to gravity.

As a result, the uncertainty in focal position for this test is under $+/-1.1$ millimeters for each test source image. Additional statistical understanding and SI-to-SI differences are captured with the additional 4 test source images. An $\mathrm{f} / 20$ beam corresponds to $70 \mathrm{~nm}$ wavefront error per millimeter of defocus. Given most of the sources emit a 2.1 micrometer wavelength, the $1.1 \mathrm{~mm}$ uncertainty equates to $1 / 27^{\text {th }}$ wave resolution.

\subsection{Tilt ( rotation about the $x$ and $y$ axes )}

Tilt between the ISIM and the OTE results in two effects: a linear focus change relative to field position and lateral shear between the common ISIM entrance pupil and the OTE exit pupil (Figure 2). The shear in the pupil is far more sensitive to tilt than the change in the focus. However, the ability to measure pupil shear is limited to two SIs, whereas the focus can be measured in 4 SIs. Note that with only one test image per SI, the tilt of an individual SI is not measured in the OTIS cryogenic test (nor is this measurement necessary since the object of the OTIS test is to check alignment of the aggregate ISIM relative to the OTE). The tilt of an individual SI is measured during the ISIM-only cryogenic test.

The longest separation between sources is $\sim 400 \mathrm{~mm}$ horizontally in Figure 3, and $200 \mathrm{~mm}$ vertically. With a $+/-1.1 \mathrm{~mm}$ uncertainty in focus determination, the tilt uncertainty from this measurement is $+/-5$ milliradians (mrad) and $+/-11$ mrad, respectively.

The most accurate measurement of pupil shear is the comparison between the FSM mask image and the NIRCam pinhole light array image. The pupil shear uncertainty is dominated by the variation of the NIRCam pupil wheel position in the rotational direction $(\sim+/-0.3 \mathrm{~mm})$. Another significant contributor is the uncertainty in the gravity sag. Nonetheless, the pupil shear can be determined to less than $1.3 \%$ of the pupil diameter, or $2 \mathrm{~mm}$. With $\sim 3$ meter distance from the pupil to the focal surface, this corresponds to less than $0.7 \mathrm{mrad}$ - an order of magnitude smaller than focus change method.

Alternately, the FSM mask location can be determined by the linear arrays of fiducial lights over the primary mirror as explained in section 2.5. Another advantage to this technique is that all near infrared sensitive instruments can be used to sense the edge of the FSM mask with these lights. In a normal afocal imaging mode, rather than in a pupil imaging mode, the instrument can simply be used to detect light that passes through for a particular fiducial light inside the FSM mask aperture versus all light blocked from an adjacent fiducial light outside the FSM mask aperture. The light from a point on the primary mirror pupil plane will appear evenly spread through the afocal imaging field of view.

Since the uncertainty is dominated by the NIRCam pupil wheel position uncertainty, the fiducial light method offers no gain in accuracy. The main purpose of this test is to check the alignment of the PM relative to the FSM mask. This uncertainty is dominated by the ability to define and register an edge of the Primary Mirror. Since the edge is chamfered, segmented, and viewed by a distant camera, the shear uncertainty is up to $0.5 \%$. A $0.5 \%$ shear is $30 \mathrm{~mm}$ at the PM edge, but only $0.7 \mathrm{~mm}$ at the FSM mask edge.

\section{SUMMARY}

The alignment uncertainty of the OTE optical system relative to the ISIM optical system is summarized for the 6 degrees of freedom in the table below. The translated and clocked positions are determined at the intersecting focal surface. The tilt of the systems about the focal surface results in shear of the intersecting pupil surfaces.

\begin{tabular}{|c|c|c|c|c|}
\hline Method & Image WFE & \multicolumn{2}{|c|}{ Image Centroiding } & Pupil Shear \\
\hline $\begin{array}{l}\text { Degree of } \\
\text { Freedom }\end{array}$ & Focus & De-center ( $\mathrm{x}$ and $\mathrm{y}$ ) & Clocked angle & Tilt (about $\mathrm{x}$ and $\mathrm{y}$ ) \\
\hline Uncertainty & $\pm 1.1 \mathrm{~mm}$ & $\pm 0.33 \mathrm{~mm}$ & $\pm 1.5 \mathrm{mrad}$ & $\pm 0.7 \mathrm{mrad}$ \\
\hline
\end{tabular}

22 CFR 125.4 (b)(13) applicable. 


\section{ACKNOWLEDGEMENTS}

The JWST Test team was supported by the JWST contract NNG11FD64C with NASA GSFC. The JWST system is a collaborative effort involving NASA, ESA, CSA, the Astronomy community and numerous principal investigators. The AOS-to-ISIM alignment test plan was accomplished through the efforts of individuals at ITT Geospatial Systems, NASA GSFC, Northrop Grumman Aerospace Systems , and Ball Aerospace and Technologies Corp. The AOS-to-ISIM alignment test plan has been vetted with several independent review teams.

\section{REFERENCES}

[1] jwst.nasa.gov

[2] Whitman, T., et. Al., " Non-invasive Optical End-to-End Test of a Large TMA Telescope (JWST) from the Intermediate Focus," Proc. SPIE 7436 0E (2009). 